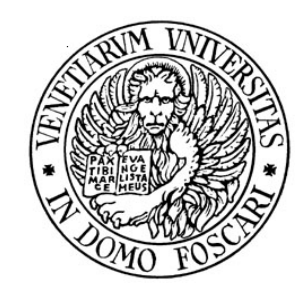

\author{
UNIVERSITÀ "CA' FOSCARI" DI VENEZIA \\ DIPARTIMENTO DI MATEMATICA APPLICATA
}

Antonella Basso - Stefania Funari

Measuring the performance of ethical mutual funds: a DEA approach

n. $107 / 2002$ 


\title{
Measuring the performance of ethical mutual funds: a DEA approach
}

\author{
Antonella Basso*, Stefania Funari \\ Department of Applied Mathematics, University of Venice \\ Dorsoduro 3825/E, 30123 Venezia, Italy \\ basso@unive.it,funari@unive.it
}

\begin{abstract}
The ethical constraints forced on an investment fund satisfy the fulfilment of humanitarian aims but may lower the investment profitability. Hence, when we measure the performance of ethical mutual funds we cannot disregard the ethical component.

In this contribution we propose a performance indicator which considers the expected return, the investment risk, the ethical component and the subscription and redemption costs together. The performance measure proposed is obtained using a DEA approach, which allows to measure the relative efficiency of decision making units in presence of a multiple input-multiple output structure.

The DEA performance indicator for ethical funds can be computed with different models, according to the nature of the ethical indicator which characterizes the socially responsible funds. In particular, a DEA model with exogenously fixed output variables and a categorical variable model seem appropriate.
\end{abstract}

Keywords: Finance; Data envelopment analysis; Mutual fund performance indexes; Ethical funds

\section{Introduction}

Ethical or socially responsible investments are playing an increasing role among the financial investments, thanks to their ability to marry profitability and social engagement together.

One of the main instruments of ethical finance is given by ethical mutual funds. These mutual funds allow investors to impose some constraints to the fund management, based on ethical principles. For example, a "socially responsible" constraint of this kind can be represented by the engagement to refrain from buying shares of companies involved in the weapon industry or in polluting activities. Other ethical objectives which can be pursued by ethical mutual funds are the engage to support non profit organizations, philanthropic institutions or special social activities.

Of course, the imposition of ethical constraints, while satisfying the fulfilment of humanitarian aims, may well lower the overall profitability of the investment.

* Partially supported by M.U.R.S.T., Research program of national interest "Models for the management of financial, insurance and operations risks". 
Therefore, when we analyze the performance of ethical mutual funds we cannot disregard the ethical component and take into account solely the portfolio return and risk.

However, the traditional numerical indexes used to measure the performance of mutual funds do not allow to take account of the ethical aspect. In particular, the Sharpe ratio (Sharpe, 1966), the reward-to-half-variance index (Ang and Chua, 1979) and the Treynor index (Treynor, 1965) are computed as ratios between the expected excess return and a risk indicator and cannot consider additional features, neither can Jensen index (Jensen, 1968).

In this contribution we try to define a performance indicator which considers simultaneously the various aspects of the portfolio performance, namely the investment return and risk, on the one hand, and the ethical behaviour on the other hand. Moreover, the performance measure proposed can take into account also the subscription and redemption costs, which contribute to determine the overall performance of the investment.

The performance measure proposed is obtained using a data envelopment analysis (DEA) approach, which is an operational research methodology introduced by Charnes, Cooper and Rhodes $(1978,1979)$ that allows to measure the relative efficiency of decision making units in presence of a multiple inputmultiple output structure.

Recently the DEA technique has been geared to the problem of mutual fund performance evaluation by Murthi, Choi and Desai (1997) and Basso and Funari (2001). The DEA mutual fund performance indexes obtained by Murthi, Choi and Desai and Basso and Funari permit to compare the investment fund efficiency not only on the basis of the fund return and risk, but also on the basis of the investment costs (subscription costs and redemption fees). In addition, different risk measures can be taken into account together; it is also possible to include in the analysis a stochastic dominance indicator that reflects the investor's preferences and the occurrence of the returns (Basso and Funari, 2001).

Another application of the philosophy of the DEA approaches to the mutual fund performance appraisal is proposed by Morey and Morey (1999) to simultaneously consider the fund risks and returns measured over different time horizons.

A first direct generalization of the DEA mutual fund performance indexes proposed in the literature suggests to exploit the multiple input-multiple output structure of a DEA model by adding among the outputs the ethical level indicator. Of course, in order to be able to make such an addition one must have at his disposal a rating ethical indicator which measures the social responsibility level.

However, the nature of the ethical indicator may often be such that a basic DEA model is not appropriate. In particular, this is the case when the ethical level is exogenously fixed and, therefore, is not under the control of managers. The same happens when the ethical indicator assigns to each fund an integer number or a character label which identifies the ethical category; in this case the ethical level represents a non-controllable categorical variable which may take a discrete set of values put on an ordinal scale. 
Hence, two additional DEA models are presented in the paper which enable to deal with the particular nature of the ethical indicator. The first model is appropriate in the case of an exogenously fixed ethical level, whereas the second one assumes that the ethical level represents a categorical variable.

If we do not have at our disposal an indicator of the ethical level of mutual funds, finally, it is still possible to define a DEA model for the valuation of the performance of mutual funds which at least takes into account the binary information on the ethical/non-ethical nature of a fund.

Lastly, in order to test the operational effectiveness and compare the differences in the performance measures computed with the various models proposed we have carried out an empirical analysis on a set of randomly generated mutual funds.

The paper is organized as follows. Section 2 introduces the DEA technique and the basic DEA model. In Section 3 we summarize the DEA measures for the mutual fund performance evaluation proposed in the literature. Sections 4 to 6 present the different DEA models for the performance evaluation of ethical mutual funds. In particular, Section 4 introduces the basic generalization which considers the ethical measure as an additional output, Section 5 presents the model for the case in which the ethical level is considered as exogenously fixed and Section 6 presents the DEA categorical models for ethical mutual funds, both in its binary and multiple categories cases. Section 7 discusses the results of the empirical application and, finally, Section 8 summarizes some concluding remarks. An Appendix reports the proofs of the theoretical results concerning the various models presented.

\section{The basic DEA model}

Let us introduce the basic DEA model originally proposed by Charnes, Cooper and Rhodes (1978). This model focuses on the analysis of the relative efficiency of a set of decision making units which may represent schools, universities, hospitals, public agencies, banks, stores and so on. All these units require some inputs and in return supply some outputs.

Let us introduce the following notation:

$j=1,2, \ldots, n \quad$ decision making units

$r=1,2, \ldots, t \quad$ outputs

$i=1,2, \ldots, m$ inputs

$y_{r j} \quad$ amount of output $r$ for unit $j$

$x_{i j} \quad$ amount of input $i$ for unit $j$

$u_{r} \quad$ weight assigned to output $r$

$v_{i} \quad$ weight assigned to input $i$.

The DEA efficiency measure is essentially defined as the ratio of a weighted sum of outputs to a weighted sum of inputs. Charnes, Cooper and Rhodes's idea is to define the efficiency measure by assigning to each decision making unit the most favourable weights. These optimal weights are computed by maximizing the efficiency ratio of the unit considered, provided that the efficiency ratios of all 
units, computed with the same weights, have an upper bound (usually set equal to 1 ).

Therefore, the DEA efficiency measure for the decision making unit $j_{0} \in$ $\{1,2, \ldots, n\}$ can be found by solving the following optimization problem

$$
\max _{\left\{v_{i}, u_{r}\right\}} \frac{\sum_{r=1}^{t} u_{r} y_{r j_{0}}}{\sum_{i=1}^{m} v_{i} x_{i j_{0}}}
$$

subject to

$$
\begin{aligned}
& \frac{\sum_{r=1}^{t} u_{r} y_{r j}}{\sum_{i=1}^{m} v_{i} x_{i j}} \leq 1 \quad j=1,2, \ldots, n \\
& u_{r} \geq \varepsilon \quad r=1,2, \ldots, t \\
& v_{i} \geq \varepsilon \quad i=1,2, \ldots, m,
\end{aligned}
$$

where $\varepsilon$ is a convenient small positive number - formally, a non-archimedean constant - that prevents the weights from vanishing (see Charnes, Cooper and Rhodes, 1979, and Charnes, Cooper, Lewin and Seiford, 1994).

The optimal objective function value (2.1) is taken as the efficiency measure assigned to unit $j_{0}$. Of course, to find the efficiency measures of the other decision making units we have to solve similar problems, targeted on each unit in turn.

Note that the efficiency measure have an upper bound of 1 , which will be reached only by the most efficient units.

The DEA model (2.1)-(2.2) is written as a fractional linear programming problem but can be conveniently converted into an equivalent linear programming problem. By letting $\sum_{i=1}^{m} v_{i} x_{i j_{0}}=1$ we obtain the so called input-oriented CCR (Charnes, Cooper and Rhodes) linear model

$$
\max \sum_{r=1}^{t} u_{r} y_{r j_{0}}
$$

subject to

$$
\begin{aligned}
& \sum_{i=1}^{m} v_{i} x_{i j_{0}}=1 \\
& \sum_{r=1}^{t} u_{r} y_{r j}-\sum_{i=1}^{m} v_{i} x_{i j} \leq 0 \quad j=1,2, \ldots, n \\
& -u_{r} \leq-\varepsilon \quad \quad r=1,2, \ldots, t \\
& -v_{i} \leq-\varepsilon \quad i=1,2, \ldots, m .
\end{aligned}
$$

The dual of problem (2.3)-(2.4) permits to identify a set of efficient units, called peer units, which are efficient with the inefficient unit's weights. These peer units are associated with the (strictly) positive basic multipliers in the optimal solution and provide a target for unit $j_{0}$ (see for example Boussofiane, Dyson and Thanassoulis, 1991). 
Alternatively, we may set $\sum_{r=1}^{t} u_{r} y_{r j_{0}}=1$, thus obtaining the so called output-oriented CCR linear model

$$
\min \sum_{i=1}^{m} v_{i} x_{i j_{0}}
$$

subject to

$$
\begin{array}{ll}
\sum_{r=1}^{t} u_{r} y_{r j_{0}}=1 & \\
\sum_{i=1}^{m} v_{i} x_{i j}-\sum_{r=1}^{t} u_{r} y_{r j} \geq 0 & j=1,2, \ldots, n \\
u_{r} \geq \varepsilon & r=1,2, \ldots, t \\
v_{i} \geq \varepsilon & i=1,2, \ldots, m .
\end{array}
$$

\section{DEA measures for mutual fund performance}

The DEA technique has recently been used to evaluate the performance of mutual funds by Murthi, Choi and Desai (1997), Morey and Morey (1999) and Basso and Funari (2001). In fact, the DEA methodology may be used to define mutual fund performance indexes that take into account several inputs, in particular different risk measures and subscription and redemption costs.

The investment cost is a relevant aspect in measuring the performance of an investment. Nevertheless, the traditional numerical indexes widely used in the literature do not allow to take into account the subscription and redemption costs usually required by an investment in mutual fund portfolios. This is the case, for example, if we consider the reward-to-volatility ratio (Sharpe, 1966), the rewardto-half-variance index (Ang and Chua, 1979) and the reward-to-variability ratio (Treynor, 1965), which are all ratios between the expected excess return and a different risk indicator; the same happens with the Jensen index (Jensen, 1968) that measures the portfolio performance by means of the intercept of a linear regression in the C.A.P.M. framework.

A first mutual fund efficiency measure which applies the DEA methodology is the DPEI index developed by Murthi, Choi and Desai (1997); it considers the mutual fund return as output and the standard deviation and transaction costs as inputs, together with operational expenses, management fees, markets and administrative expenses and a trading turnover ratio.

The $I_{D E A-1}$ index proposed by Basso and Funari (2001) can be seen, in a sense, as a generalization of DPEI which allows to consider different risk measures, though there exist slight differences with regard to the transaction costs taken into consideration. In particular, $I_{D E A-1}$ takes into account only the subscription costs and redemption fees that directly burden the investors but not the other expenses that have already been deducted from the fund quotations. 
Let us consider a set of $n$ mutual funds $j=1,2, \ldots, n$ with risky returns $R_{j}$ and introduce the following notation:

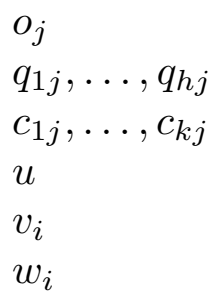

a return measure of fund $j$

$h$ risk measures for fund $j$

$k$ subscription and/or redemption costs for fund $j$ weight assigned to the return measure $o_{j}$

weight assigned to the risk measure $i(i=1,2, \ldots, h)$

weight assigned to the cost $i(i=1,2, \ldots, k)$.

As output, the model considers a return measure, which can be either the expected return $E\left(R_{j}\right)$ or the expected excess return $E\left(R_{j}\right)-\delta$, where $\delta$ is the riskless rate of return.

As inputs, it considers some risk measures and the subscription and redemption costs. In particular, among the risk measures one can include the standard deviation of the return $\sigma_{j}=\sqrt{\operatorname{Var}\left(R_{j}\right)}$, the root of the halfvariance risk indicator $H V_{j}=E\left(\min \left[R_{j}-E\left(R_{j}\right), 0\right]\right)^{2}$, and the $\beta$ coefficient $\beta_{j}=\operatorname{Cov}\left(R_{j}, R_{m}\right) / \operatorname{Var}\left(R_{m}\right)$, where $R_{m}$ denotes the market portfolio return.

The DEA performance index of mutual fund investments for fund $j_{0}$, $I_{j_{0}, D E A-1}$, is computed as the optimal objective function value of the following DEA problem

$$
\max _{\left\{u, v_{i}, w_{i}\right\}} \frac{u o_{j_{0}}}{\sum_{i=1}^{h} v_{i} q_{i j_{0}}+\sum_{i=1}^{k} w_{i} c_{i j_{0}}}
$$

subject to

$$
\begin{array}{ll}
\frac{u o_{j}}{\sum_{i=1}^{h} v_{i} q_{i j}+\sum_{i=1}^{k} w_{i} c_{i j}} \leq 1 & j=1,2, \ldots, n \\
u \geq \varepsilon & \\
v_{i} \geq \varepsilon \quad & i=1,2, \ldots, h \\
w_{i} \geq \varepsilon \quad & i=1,2, \ldots, k .
\end{array}
$$

If we let an asterisk denotes the optimal values of the variables, we have

$$
I_{j_{0}, D E A-1}=\frac{u^{*} o_{j_{0}}}{\sum_{i=1}^{h} v_{i}^{*} q_{i j_{0}}+\sum_{i=1}^{k} w_{i}^{*} c_{i j_{0}}} .
$$

Equation (3.3) suggests that the $I_{D E A-1}$ index generalizes the traditional Sharpe, Treynor and reward-to-half-variance indexes, which may be seen as ratios between the expected excess return (an output) and a different risk indicator (an input). In effect, Basso and Funari (2001) show that with the excess return as output and the standard deviation of the return as the only input considered - so that the entrance and exit investment costs are omitted - then the $I_{D E A-1}$ efficiency index coincides with the Sharpe ratio multiplied by a normalization constant which scales it off in the interval $[0,1]$. Analogously, by taking as risk measure either the root of the half-variance or the $\beta$ coefficient we get the reward-to-half-variance and the Treynor indexes, respectively. 
The DEA efficiency measure $I_{D E A-1}$ exhibits some interesting properties discussed in Basso and Funari (2001). A first property concerns the length of the investment horizon. It is known that the traditional performance indexes are sensitive to the assumed investment horizon, since we may get different results according to the data frequency, i.e. the time interval (week, month, year...) to which the portfolio returns refer. Hence, a systematic bias could be observed if the index is computed by assuming a holding period which is different from the one chosen by the investors; see Levy (1972) for the Sharpe index and Levhari and Levy (1977) for the Treynor and Jensen performance measures. On the other hand, if logarithmic returns are used and under the assumption of stationarity and independence of the returns over time, it can be shown that the DEA efficiency measure $I_{D E A-1}$ is invariant with respect to the investment horizon chosen.

Another useful property of the DEA approach follows from the solution of the dual problem, which allows to suggest to the inefficient units a "virtual unit" that they could imitate in order to improve their efficiency, as it is efficient with the inefficient unit's weights. From a financial point of view, this composite unit could be considered as a target benchmark for the inefficient fund $j_{0}$. Fund $j_{0}$ could improve its performance by trying to imitate the behaviour of the efficient composite unit, which has an input/output orientation which is similar to that of the (inefficient) fund $j_{0}$. Notice that this benchmark portfolio is a "fund of funds" which can actually be purchased (Morey and Morey, 1999).

\section{A basic DEA model for ethical funds}

A first step in order to take into account not only the risk and return (and eventually the costs) of the mutual fund investment but also the fulfillment of ethical aims, could be to define a measure of the social responsibility level. In effect, there are now some attempts to define rating indicators which divide the funds into categories on the basis of the restrictions, more or less stringent, imposed to the fund management by the ethical constraints.

Let $e_{j}$ denote the ethical measure for fund $j(j=1,2, \ldots, n)$ and let us assume that it represents a non negative real number. Then it would be easy to generalize the DEA mutual fund performance measure $I_{D E A-1}$ by adding a second output: besides the expected return measure $o_{j}$, we can easily introduce the ethical measure $e_{j}$ among the outputs. In this way we can compute the basic DEA measure for ethical mutual fund performance, $I_{D E A-E}$, for fund $j_{0}$ as follows

$$
I_{j_{0}, D E A-E}=\frac{u_{1}^{*} o_{j_{0}}+u_{2}^{*} e_{j_{0}}}{\sum_{i=1}^{h} v_{i}^{*} q_{i j_{0}}+\sum_{i=1}^{k} w_{i}^{*} c_{i j_{0}}},
$$

where $u_{1}$ is the weight assigned to the return measure $o_{j}, u_{2}$ is the weight assigned to the ethical measure $e_{j}$ and the asterisk characterizes the optimal solution of the following two-output DEA problem

$$
\max _{\left\{u_{r}, v_{i}, w_{i}\right\}} \frac{u_{1} o_{j_{0}}+u_{2} e_{j_{0}}}{\sum_{i=1}^{h} v_{i} q_{i j_{0}}+\sum_{i=1}^{k} w_{i} c_{i j_{0}}}
$$


subject to

$$
\begin{array}{ll}
\frac{u_{1} o_{j}+u_{2} e_{j}}{\sum_{i=1}^{h} v_{i} q_{i j}+\sum_{i=1}^{k} w_{i} c_{i j}} \leq 1 & j=1,2, \ldots, n \\
u_{r} \geq \varepsilon & r=1,2 \\
v_{i} \geq \varepsilon & i=1,2, \ldots, h \\
w_{i} \geq \varepsilon & i=1,2, \ldots, k .
\end{array}
$$

It is easy to see the relation which connects the one-output performance measure $I_{D E A-1}$ with the two-output measure $I_{D E A-E}$. In fact, by adding an output indicator in a DEA efficiency measurement problem we can only improve the efficiency score, so that the following proposition holds.

Proposition 1. Let $I_{j_{0}, D E A-1}$ be the DEA performance index (3.3) and $I_{j_{0}, D E A-E}$ the basic DEA measure for ethical mutual fund performance (4.1); we have

$$
I_{j_{0}, D E A-1} \leq I_{j_{0}, D E A-E}
$$

The proof of Proposition 1 is reported in the Appendix.

Moreover, it could be natural to choose an ethical measure which assigns the ethical funds a strictly positive number while the measure assigned to the nonethical funds is set equal to zero. In this case, it can be shown that the value of the ethical performance index $I_{D E A-E}$ for the non-ethical funds will coincide with the value of $I_{D E A-1}$.

Proposition 2. Let $I_{j_{0}, D E A-1}$ be the DEA performance index (3.3) and $I_{j_{0}, D E A-E}$ the basic DEA measure for ethical mutual fund performance (4.1); if $e_{j_{0}}=0$ and $e_{j} \geq 0$ for all $j$ we have

$$
I_{j_{0}, D E A-1}=I_{j_{0}, D E A-E}
$$

The proof of Proposition 2 is reported in the Appendix.

\section{An exogenously fixed output DEA model for ethical funds}

Nevertheless, the ethical level of the investments in a socially responsible fund is often chosen by investors a priori and cannot be arbitrarily modified; the output $e_{j}$ can therefore be considered as exogenously fixed, beyond the discretionary control of managers. On the other hand, the virtual unit suggested by the basic DEA technique as efficient benchmark for an inefficient unit could have an ethical level different from the one chosen by investors.

To avoid this inconvenient, when the analysis includes fixed variables (either inputs or outputs) the basic DEA model has to be conveniently modified. To this aim, Banker and Morey (1986a) suggest to keep the level of the exogenously fixed variables constant at their current value: only the value of the discretionary or controllable variables should be modified by the DEA programs. 
We may apply to the performance measurement of ethical mutual funds a model analogous to the one suggested by Banker and Morey (1986a) for exogenously fixed outputs. With respect to the original Banker and Morey's model we have only relaxed the convexity constraint, which could be overcome in the case of investment funds.

The changes suggested by Banker and Morey to keep constant the level of the non-controllable output can be applied to the dual of the linear programming problem written in an output-oriented form. We obtain the following model:

$$
\max \quad z_{0}+\varepsilon \sum_{i=1}^{h} s_{i}^{-}+\varepsilon \sum_{i=1}^{k} s_{h+i}^{-}+\varepsilon s_{1}^{+}
$$

subject to

$$
\begin{array}{rl}
\sum_{j=1}^{n} q_{i j} \lambda_{j}+s_{i}^{-}=q_{i j_{0}} & i=1,2, \ldots, h \\
\sum_{j=1}^{n} c_{i j} \lambda_{j}+s_{h+i}^{-}=c_{i j_{0}} & i=1,2, \ldots, k \\
z_{0} o_{j_{0}}-\sum_{j=1}^{n} o_{j} \lambda_{j}+s_{1}^{+}=0 & \\
-\sum_{j=1}^{n} e_{j} \lambda_{j}+s_{2}^{+} & =-e_{j 0} \\
\lambda_{j} \geq 0 \quad j & =1,2, \ldots, n \\
s_{i}^{-} \geq 0 \quad i & =1,2, \ldots, h+k \\
s_{r}^{+} \geq 0 \quad r & =1,2,
\end{array}
$$

where $z_{0}$ is the dual variable associated with the equality constraint, $\lambda_{j}$ are the dual variables associated with the mutual funds constraints and $s_{i}^{-}$and $s_{r}^{+}$are the dual variables connected with the input and output weight constraints, respectively.

The primal of the dual problem (5.1)-(5.2) leads to the following DEA fractional programming problem

$$
\max _{\left\{u_{r}, v_{i}, w_{i}\right\}} \frac{u_{1} o_{j_{0}}}{\sum_{i=1}^{h} v_{i} q_{i j_{0}}+\sum_{i=1}^{k} w_{i} c_{i j_{0}}-u_{2} e_{j_{0}}}
$$

subject to

$$
\begin{array}{ll}
\frac{u_{1} o_{j}}{\sum_{i=1}^{h} v_{i} q_{i j}+\sum_{i=1}^{k} w_{i} c_{i j}-u_{2} e_{j}} & \leq 1 \\
u_{1} \geq \varepsilon, \quad u_{2} \geq 0 \\
v_{i} \geq \varepsilon & i=1, \ldots, h \\
w_{i} \geq \varepsilon & i=1,2, \ldots, k .
\end{array}
$$


The performance measure when the ethical level is assumed uncontrollable, $I_{D E A-U}$, is given by the optimal value of the objective function (5.3) or, equivalently, by the inverse of the optimal value of $z_{0}$ in problem (5.1)-(5.2).

One can wonder the effect on the performance measure of fixing the value of the uncontrollable ethical level. In effect, it can be proved that the non-controllable case performance measure $I_{D E A-U}$ has a value less than or equal to the value of the basic DEA index $I_{D E A-E}$.

Proposition 3. Let $I_{j_{0}, D E A-U}$ be the optimal value of the DEA problem (5.3)(5.4) and $I_{j_{0}, D E A-E}$ the basic performance index (4.1); we have

$$
I_{j_{0}, D E A-U} \leq I_{j_{0}, D E A-E} .
$$

The proof of Proposition 3 is reported in the Appendix.

Moreover, it can be shown that if the non-ethical funds are assigned a null ethical measure $\left(e_{j}=0\right)$, then for the non-ethical funds the non-controllable case performance measure $I_{D E A-U}$ coincides with the value of the DEA index $I_{D E A-E}$.

Proposition 4. Let $I_{j_{0}, D E A-U}$ be the optimal value of the DEA problem (5.3)(5.4) and $I_{j_{0}, D E A-E}$ the basic performance index (4.1); if $e_{j_{0}}=0$ we have

$$
I_{j_{0}, D E A-U}=I_{j_{0}, D E A-E} .
$$

The proof of Proposition 4 is reported in the Appendix.

\section{DEA categorical models for ethical funds}

Sometimes, the rating of the socially responsible mutual funds is carried out by dividing the funds into categories of gradually increasing ethical level; the various categories are usually marked with either an integer number or a character label. In such a case, the ethical indicator cannot be considered as a real measure; it is instead a non-controllable categorical variable with a discrete set of values put on an ordinal scale.

The presence of non-controllable categorical variables does not recommend the use of a basic DEA model. The reason lies in the fact that the virtual composite unit suggested by the basic DEA technique as efficient benchmark could well have an ethical level defined as it were measured on an interval scale, with a value which has no correspondence with any of the existing categories.

Efficiency analyses in presence of non-controllable categorical variables are well known in the DEA literature. Two implementations are given by a performance analysis of pharmacies carried out by Banker and Morey (1986b), in which the pharmacies were divided into categories according to the size of their market (population served), and a study of Cooper, Seiford and Tone (2000) on the relative efficiency of the public libraries in Tokio; the libraries were classified into categories according to the kind of the area covered (business, shopping and 
residential areas; the third category has the greatest advantage while the first one is at a disadvantage).

The idea with categorical variables is to modify the basic DEA model in order to ensure that the virtual unit suggested as efficient benchmark includes only units which have the same or lower values of the categorical variables. In the ethical mutual fund analysis, the ordinal scale can be defined in such a way: the nonethical funds are considered as having an advantage, while as the ethical level increases the ethical funds are considered as more and more in a disadvantage as they have to meet more ethical requirements.

In order to exclude the funds that are "less ethical" from the peer group we can define $p-1$ binary variables, where $p$ is the number of categories $(p-1$ categories classifies the ethical funds and one indicates the non-ethical funds).

To understand the functioning of the model let us first introduce the simple binary case of two ethical categories (ethical/non-ethical funds) and let us define a binary variable $d$ as follows

$$
d_{j}= \begin{cases}0 & \text { if fund } j \text { is ethical } \\ 1 & \text { if fund } j \text { is not ethical. }\end{cases}
$$

This binary categorical case, furthermore, answers our purpose in the not infrequent case in which we do not have at our disposal an indicator of the ethical level of mutual funds but only have the binary information on the ethical/nonethical nature of a fund.

An appropriate categorical DEA model for the binary ethical/non-ethical case is

$$
\max z_{0}+\varepsilon \sum_{i=1}^{h} s_{i}^{-}+\varepsilon \sum_{i=1}^{k} s_{h+i}^{-}+\varepsilon s_{1}^{+}
$$

subject to

$$
\begin{aligned}
& \sum_{j=1}^{n} q_{i j} \lambda_{j}+s_{i}^{-}=q_{i j_{0}} \quad i=1,2, \ldots, h \\
& \sum_{j=1}^{n} c_{i j} \lambda_{j}+s_{h+i}^{-}=c_{i j_{0}} \quad i=1,2, \ldots, k \\
& z_{0} o_{j_{0}}-\sum_{j=1}^{n} o_{j} \lambda_{j}+s_{1}^{+}=0 \\
& \sum_{j=1}^{n} \frac{q_{1 j}}{q_{1 j_{0}}} d_{j} \lambda_{j} \leq d_{j_{0}} \\
& \lambda_{j} \geq 0 \quad j=1,2, \ldots, n \\
& s_{i}^{-} \geq 0 \quad i=1,2, \ldots, h+k \\
& s_{1}^{+} \geq 0 .
\end{aligned}
$$

Observe that the binary categorical model (6.2)-(6.3) is similar to the noncontrollable output model (5.1)-(5.2): the only difference is given by the constraint 
on the ethical measure $e_{j},-\sum_{j=1}^{n} e_{j} \lambda_{j}+s_{2}^{+}=-e_{j_{0}}$, which is substituted with a constraint involving the ethical categorical variable $d_{j}$

$$
\sum_{j=1}^{n} \frac{q_{1 j}}{q_{1 j_{0}}} d_{j} \lambda_{j} \leq d_{j_{0}}
$$

Let us analyze in detail this constraint. For the non-ethical funds $\left(d_{j_{0}}=1\right)$ constraint (6.4) is redundant since

$$
\sum_{j=1}^{n} \frac{q_{1 j}}{q_{1 j_{0}}} d_{j} \lambda_{j} \leq \sum_{j=1}^{n} \frac{q_{1 j}}{q_{1 j_{0}}} \lambda_{j} \leq 1
$$

where the last inequality follows from the first of constraints (6.3). Of course, in order to write constraint (6.4) for any fund $j_{0}$ the first risk measure must be strictly positive $\left(q_{1 j}>0\right.$ for all $\left.j\right)$. On the contrary, for the ethical funds $\left(d_{j_{0}}=0\right)$ constraint (6.4) requires that the multipliers associated to the non-ethical funds are zero $\left(\lambda_{j}=0\right.$ if $\left.d_{j}=1\right)$. Note that if the additional convexity constraint $\sum_{j=1}^{n} \lambda_{j}=1$ is imposed, as in Banker and Morey (1986b), then constraint (6.4) is equivalent to the simpler inequality $\sum_{j=1}^{n} d_{j} \lambda_{j} \leq d_{j_{0}}$.

If the mutual funds are classified into more than two ethical categories, as is the case, for example, if we consider non-ethical funds and ethical funds with social responsibility levels 1 (low), 2 (average) and 3 (high), a single ethical constraint is not sufficient. Let us define three binary variables $d^{(1)}, d^{(2)}, d^{(3)}$ as follows

Fund category $\quad d_{j}^{(1)} \quad d_{j}^{(2)} \quad d_{j}^{(3)}$

Non-ethical

Ethical level 1

Ethical level 2

Ethical level 3

$\begin{array}{lll}1 & 1 & 1 \\ 1 & 1 & 0 \\ 1 & 0 & 0 \\ 0 & 0 & 0\end{array}$

The categorical DEA model for multiple ethical categories requires the introduction of 3 (in general, $p-1$ ) ethical constraints and can be written as

$$
\max z_{0}+\varepsilon \sum_{i=1}^{h} s_{i}^{-}+\varepsilon \sum_{i=1}^{k} s_{h+i}^{-}+\varepsilon s_{1}^{+}
$$


subject to

$$
\begin{array}{ll}
\sum_{j=1}^{n} q_{i j} \lambda_{j}+s_{i}^{-}=q_{i j_{0}} & i=1,2, \ldots, h \\
\sum_{j=1}^{n} c_{i j} \lambda_{j}+s_{h+i}^{-}=c_{i j_{0}} & i=1,2, \ldots, k \\
z_{0} o_{j_{0}}-\sum_{j=1}^{n} o_{j} \lambda_{j}+s_{1}^{+}=0 & \\
\sum_{j=1}^{n} \frac{q_{1 j}}{q_{1 j_{0}}} d_{j}^{(a)} \lambda_{j} \leq d_{j_{0}}^{(a)} & a=1,2,3 \\
\lambda_{j} \geq 0 \quad j=1,2, \ldots, n & \\
s_{i}^{-} \geq 0 \quad i=1,2, \ldots, h+k \\
s_{1}^{+} \geq 0 .
\end{array}
$$

Let us point out that with the categorical models (6.2)-(6.3) and (6.6)-(6.7) we can still have virtual units with an ethical level which does not correspond to any category, but an ethical mutual fund is now compared only to funds which are at least socially responsible as itself, and this should give rise to a fairer evaluation process.

We will denote the performance measure for an ethical categorical variable by $I_{D E A-C}$ in the multiple categories case and $I_{D E A-B}$ in the binary ethical/nonethical case; this performance measure is computed as the inverse of the optimal value of $z_{0}$ of problem (6.6)-(6.7) or (6.2)-(6.3), respectively.

An interesting property of invariance of the efficiency measure can be shown for the non-ethical funds.

Proposition 5. Let $I_{j_{0}, D E A-C}$ be the inverse of the optimal value of $z_{0}$ in the DEA problem (6.6)-(6.7), $I_{j_{0}, D E A-U}$ the optimal value of the DEA problem (5.3)(5.4) and $I_{j_{0}, D E A-E}$ the basic performance index (4.1); if $e_{j_{0}}=0$ and $d_{j}^{(a)}=1 \forall a$ we have

$$
I_{j_{0}, D E A-C}=I_{j_{0}, D E A-U}=I_{j_{0}, D E A-E}
$$

The proof of Proposition 5 is reported in the Appendix.

As concerns the comparison between the binary and the multiple categories cases, we can state the following result.

Proposition 6. Let $I_{j_{0}, D E A-B}$ and $I_{j_{0}, D E A-C}$ be the inverse of the optimal value of $z_{0}$ in the DEA problems (6.2)-(6.3) and (6.6)-(6.7), respectively; then

$$
I_{j_{0}, D E A-B} \leq I_{j_{0}, D E A-C}
$$

moreover, for the non-ethical funds we have

$$
I_{j_{0}, D E A-B}=I_{j_{0}, D E A-C}
$$


The proof of Proposition 6 is reported in the Appendix.

\section{An empirical application}

In order to test the applicability of the different DEA models proposed to treat the measurement of the performance of ethical mutual funds, we have applied these models to a set of 50 randomly generated mutual funds.

The inputs considered in the empirical analysis are three brackets of subscription costs (per cent subscription cost relative to 5000,25000 and 50000 dollars of initial investment), three brackets of redemption costs (per cent redemption cost assuming a length of investment period of 1, 2 and 3 years, respectively) and two risk measures given by the standard deviation of the fund return $\sigma_{j}$ and the $\beta$ coefficient.

The two outputs considered are the expected return $E\left(R_{j}\right)$ and an ethical indicator $e_{j}$ which takes integer values in the range 0 to 3: a 0 level marks the non-ethical funds while a strictly positive integer denotes the ethical level of the socially responsible funds. The one-output DEA model (3.1)-(3.2) disregards the ethical indicator $e_{j}$, in the basic DEA model (4.2)-(4.3) and in the exogenously fixed output model (5.1)-(5.2) it represents the ethical measure and in the DEA categorical model (6.6)-(6.7) it is considered as a categorical variable. For the binary categorical model (6.2)-(6.3) the ethical variable $d_{j}$ has been defined as $d_{j}=0$ if $e_{j}=0$ and $d_{j}=1$ if $e_{j}>0$.

The first 30 funds are non-ethical, whereas the last 20 have an ethical nature as follows: 6 funds have ethical measure 1, 7 have ethical measure 2 and 7 have ethical measure 3. The values of the other input and output variables of each fund have been generated with a Monte Carlo technique either as a normal or as a uniform random variable, on a likely basis which simulates the behaviour of a real market.

Table 1 shows the performance measures $I_{D E A-1}, I_{D E A-E}, I_{D E A-U}$, $I_{D E A-C}, I_{D E A-B}$ obtained for the 50 mutual funds analyzed, together with the consequent relative ranking.

We know from Propositions 1-6 that the performance measures $I_{D E A-1}$, $I_{D E A-E}, I_{D E A-U}, I_{D E A-C}, I_{D E A-B}$ for a non-ethical fund coincide while for the ethical funds we only know that both $I_{D E A-1}$ and $I_{D E A-U}$ are less than or equal to $I_{D E A-E}$ while $I_{D E A-B} \leq I_{D E A-C}$. The results displayed in Table 1 highlight these properties; moreover, for the ethical funds the empirical results show that any relation can hold between $I_{D E A-C}$ and $I_{D E A-E}$, on the one hand, and between $I_{D E A-U}$ and $I_{D E A-C}$ on the other hand. In fact, from Table 1 we observe the following inequalities for the ethical funds F39, F46 and F47

$$
\begin{aligned}
& I_{39, D E A-U}<I_{39, D E A-B}<I_{39, D E A-C}<I_{39, D E A-E} \\
& I_{46, D E A-B}<I_{46, D E A-C}<I_{46, D E A-U}=I_{46, D E A-E} \\
& I_{47, D E A-B}<I_{47, D E A-U}<I_{47, D E A-E}<I_{47, D E A-C} .
\end{aligned}
$$


Table 1 Empirical results obtained for 50 simulated mutual funds; the first 30 funds are not ethical, the last 20 are ethical funds. The table reports the performance measure obtained with the different DEA models presented in the paper and the relative ranking (in italics).

\begin{tabular}{|c|c|c|c|c|c|c|c|c|c|c|c|}
\hline \multirow{2}{*}{$\begin{array}{l}\text { Funds } \\
\text { F1 }\end{array}$} & \multirow{2}{*}{$\begin{array}{c}\begin{array}{c}\text { Ethical } \\
\text { level }\end{array} \\
0\end{array}$} & \multicolumn{2}{|c|}{$I_{D E A-1}$} & \multicolumn{2}{|c|}{$I_{D E A-E}$} & \multicolumn{2}{|c|}{$I_{D E A-U}$} & \multicolumn{2}{|c|}{$I_{D E A-C}$} & \multicolumn{2}{|c|}{$I_{D E A-B}$} \\
\hline & & 0.804 & 12 & 0.805 & 20 & 0.805 & 17 & 0.805 & 15 & 0.805 & 14 \\
\hline $\mathrm{F} 2$ & 0 & 0.104 & 47 & 0.104 & 50 & 0.104 & 49 & 0.104 & 48 & 0.104 & 48 \\
\hline F3 & 0 & 0.480 & 30 & 0.480 & 38 & 0.480 & 33 & 0.480 & 32 & 0.480 & 31 \\
\hline $\mathrm{F} 4$ & 0 & 0.971 & 6 & 0.971 & 14 & 0.971 & 11 & 0.971 & 12 & 0.971 & 11 \\
\hline F5 & 0 & 1.000 & 1 & 1.000 & 1 & 1.000 & 1 & 1.000 & 1 & 1.000 & 1 \\
\hline $\mathrm{F} 6$ & 0 & 0.613 & 23 & 0.613 & 32 & 0.613 & 26 & 0.613 & 25 & 0.613 & 24 \\
\hline F7 & 0 & 0.907 & 9 & 0.907 & 15 & 0.907 & 12 & 0.907 & 13 & 0.907 & 12 \\
\hline F8 & 0 & 0.729 & 16 & 0.729 & 25 & 0.729 & 19 & 0.729 & 19 & 0.729 & 18 \\
\hline F9 & 0 & 0.705 & 17 & 0.705 & 27 & 0.705 & 21 & 0.705 & 21 & 0.705 & 20 \\
\hline F10 & 0 & 0.569 & 25 & 0.569 & 34 & 0.569 & 28 & 0.569 & 28 & 0.569 & 28 \\
\hline F11 & 0 & 0.684 & 18 & 0.684 & 28 & 0.684 & 23 & 0.684 & 22 & 0.684 & 21 \\
\hline F12 & 0 & 0.455 & 31 & 0.455 & 39 & 0.455 & 34 & 0.455 & 33 & 0.455 & 32 \\
\hline F13 & 0 & 1.000 & 1 & 1.000 & 1 & 1.000 & 1 & 1.000 & 1 & 1.000 & 1 \\
\hline F14 & 0 & 0.557 & 26 & 0.557 & 36 & 0.557 & 30 & 0.557 & 29 & 0.557 & 29 \\
\hline F15 & 0 & 0.452 & 32 & 0.452 & 40 & 0.452 & 35 & 0.452 & 34 & 0.452 & 33 \\
\hline F16 & 0 & 0.403 & 37 & 0.403 & 44 & 0.403 & 40 & 0.403 & 39 & 0.403 & 38 \\
\hline F17 & 0 & 0.154 & 44 & 0.154 & 48 & 0.154 & 47 & 0.154 & 46 & 0.154 & 45 \\
\hline F18 & 0 & 0.118 & 46 & 0.118 & 49 & 0.118 & 48 & 0.118 & 47 & 0.118 & 47 \\
\hline F19 & 0 & 0.759 & 15 & 0.759 & 24 & 0.759 & 18 & 0.759 & 17 & 0.759 & 16 \\
\hline F20 & 0 & 0.285 & 41 & 0.285 & 47 & 0.285 & 44 & 0.285 & 42 & 0.285 & 41 \\
\hline F21 & 0 & 0.407 & 35 & 0.407 & 42 & 0.407 & 37 & 0.407 & 36 & 0.407 & 35 \\
\hline F22 & 0 & 0.411 & 34 & 0.411 & 41 & 0.411 & 36 & 0.411 & 35 & 0.411 & 34 \\
\hline F23 & 0 & 0.405 & 36 & 0.405 & 43 & 0.405 & 38 & 0.405 & 37 & 0.405 & 36 \\
\hline F24 & 0 & 0.571 & 24 & 0.571 & 33 & 0.571 & 27 & 0.571 & 27 & 0.571 & 27 \\
\hline F25 & 0 & 0.883 & 10 & 0.883 & 18 & 0.883 & 14 & 0.883 & 14 & 0.883 & 13 \\
\hline F26 & 0 & 0.330 & 38 & 0.330 & 46 & 0.330 & 43 & 0.330 & 41 & 0.330 & 40 \\
\hline F27 & 0 & 0.652 & 20 & 0.652 & 30 & 0.652 & 24 & 0.652 & 23 & 0.652 & 22 \\
\hline F28 & 0 & 0.527 & 27 & 0.527 & 37 & 0.527 & 32 & 0.527 & 30 & 0.527 & 30 \\
\hline F29 & 0 & 1.000 & 1 & 1.000 & 1 & 1.000 & 1 & 1.000 & 1 & 1.000 & 1 \\
\hline F30 & 0 & 1.000 & 1 & 1.000 & 1 & 1.000 & 1 & 1.000 & 1 & 1.000 & 1 \\
\hline F31 & 1 & 0.649 & 21 & 0.673 & 29 & 0.649 & 25 & 0.712 & 20 & 0.712 & 19 \\
\hline F32 & 1 & 0.794 & 13 & 0.887 & 17 & 0.876 & 15 & 1.000 & 1 & 1.000 & 1 \\
\hline F33 & 1 & 0.809 & 11 & 0.895 & 16 & 0.885 & 13 & 1.000 & 1 & 1.000 & 1 \\
\hline F34 & 1 & 0.635 & 22 & 0.707 & 26 & 0.684 & 22 & 0.783 & 16 & 0.783 & 15 \\
\hline F35 & 1 & 0.065 & 49 & 0.339 & 45 & 0.070 & 50 & 0.089 & 49 & 0.089 & 49 \\
\hline F36 & 1 & 0.522 & 28 & 0.562 & 35 & 0.539 & 31 & 0.637 & 24 & 0.637 & 23 \\
\hline F37 & 2 & 0.307 & 40 & 0.646 & 31 & 0.348 & 42 & 0.383 & 40 & 0.383 & 39 \\
\hline F38 & 2 & 0.170 & 43 & 0.778 & 22 & 0.187 & 46 & 0.203 & 44 & 0.203 & 43 \\
\hline F39 & 2 & 0.419 & 33 & 0.793 & 21 & 0.564 & 29 & 0.600 & 26 & 0.594 & 26 \\
\hline F40 & 2 & 0.925 & 8 & 1.000 & 1 & 1.000 & 1 & 1.000 & 1 & 1.000 & 1 \\
\hline F41 & 2 & 0.674 & 19 & 0.770 & 23 & 0.713 & 20 & 0.743 & 18 & 0.743 & 17 \\
\hline F42 & 2 & 1.000 & 1 & 1.000 & 1 & 1.000 & 1 & 1.000 & 1 & 1.000 & 1 \\
\hline F43 & 2 & 0.122 & 45 & 0.838 & 19 & 0.192 & 45 & 0.171 & 45 & 0.170 & 44 \\
\hline F44 & 3 & 0.102 & 48 & 0.991 & 12 & 0.352 & 41 & 0.253 & 43 & 0.142 & 46 \\
\hline F45 & 3 & 0.040 & 50 & 1.000 & 1 & 1.000 & 10 & 0.089 & 50 & 0.045 & 50 \\
\hline F46 & 3 & 0.206 & 42 & 1.000 & 1 & 1.000 & 1 & 0.503 & 31 & 0.284 & 42 \\
\hline F47 & 3 & 0.503 & 29 & 0.973 & 13 & 0.845 & 16 & 1.000 & 1 & 0.596 & 25 \\
\hline F48 & 3 & 0.324 & 39 & 1.000 & 1 & 0.405 & 39 & 0.405 & 38 & 0.405 & 37 \\
\hline F49 & 3 & 0.959 & 7 & 1.000 & 1 & 1.000 & 1 & 1.000 & 1 & 1.000 & 1 \\
\hline F50 & 3 & 0.777 & 14 & 1.000 & 1 & 1.000 & 1 & 0.980 & 11 & 0.980 & 10 \\
\hline
\end{tabular}


Table 2 Correlation coefficients between the performance measures obtained for the 20 simulated ethical mutual funds with the different DEA models.

\begin{tabular}{llllll}
\hline & $I_{D E A-1}$ & $I_{D E A-E}$ & $I_{D E A-U}$ & $I_{D E A-C}$ & $I_{D E A-B}$ \\
\hline$I_{D E A-1}$ & 1.000 & 0.273 & 0.664 & 0.941 & 0.985 \\
$I_{D E A-E}$ & & 1.000 & 0.636 & 0.352 & 0.256 \\
$I_{D E A-U}$ & & & 1.000 & 0.727 & 0.655 \\
$I_{D E A-C}$ & & & 1.000 & 0.957 \\
$I_{D E A-B}$ & & & & 1.000 \\
\hline
\end{tabular}

Table 3 Correlation coefficients between the relative ranking of all the 50 simulated mutual funds computed with the different DEA models.

\begin{tabular}{llllll}
\hline & $I_{D E A-1}$ & $I_{D E A-E}$ & $I_{D E A-U}$ & $I_{D E A-C}$ & $I_{D E A-B}$ \\
\hline$I_{D E A-1}$ & \multirow{2}{*}{1.000} & 0.549 & 0.820 & 0.935 & 0.974 \\
$I_{D E A-E}$ & & 1.000 & 0.811 & 0.640 & 0.591 \\
$I_{D E A-U}$ & & & 1.000 & 0.863 & 0.829 \\
$I_{D E A-C}$ & & & 1.000 & 0.970 \\
$I_{D E A-B}$ & & & & 1.000 \\
\hline
\end{tabular}

Another feature pointed out by the empirical results is that, as one can expect, $I_{D E A-1}$ gives the minimum efficiency score.

In order to verify how significant the differences among the various performance measures are, Table 2 reports the values of the correlation coefficients computed among the performance measures $I_{D E A-1}, I_{D E A-E}, I_{D E A-U}, I_{D E A-C}$, $I_{D E A-B}$ for all ethical funds. The correlation coefficients indicate that the efficiency results obtained with the exogenously fixed output and the categorical models are more correlated with the one-output model results than with the results of the basic DEA model for ethical funds. Apparently, the basic two-output model (4.2)-(4.3) gives much emphasis on the ethical level, thus raising the efficiency score of the ethical funds maybe too much. As expected, the correlation between the binary and the multiple categories models is very high.

Sometimes, one is interested in the relative ranking more than in the performance value in itself; on the other hand, the rating of a fund may vary even when the performance measure does not change while, in other cases, we may observe the same rating even if the efficiency score is slightly modified. Table 3 presents the correlation coefficients among the relative rankings obtained with the different DEA models for all the 50 funds analyzed. Although softened, the differences pointed out in Table 2 can in substance be observed also in Table 3. 


\section{Concluding remarks}

We have proposed a few models which apply the DEA technique to the measurement of the performance of ethical mutual funds. The models differ in the nature of the ethical indicator, which can be either a measure of the ethical level of the fund or a categorical variable. Moreover, the ethical level can sometimes be considered as exogenously fixed and beyond the control of managers, and this constraint has to be taken into account by the measurement procedure. Besides the basic DEA model for ethical mutual fund performance measurement, we attain two special models which take into account either the categorical nature of the ethical level or the fact that the ethical level is fixed in advance and cannot be modified. Finally, a special binary ethical/non-ethical case gives a peculiar categorical model.

The various models presented have been compared both from a theoretical point of view and through an empirical analysis.

Of course, all these models can naturally be modified or extended by considering other input or output variables, which allow to take account of other aspects that may be useful in the comparison of ethical mutual funds.

\section{Appendix}

We report in this Appendix an outline of the proofs of Propositions 1-6.

\section{Proof of Proposition 1}

Proposition 1 can be proved by comparing the constraints of the dual problems of the input oriented linear programming problem equivalent to the DEA problems (3.1)-(3.2) and (4.2)-(4.3): the constraints of the first one are a subset of the constraints of the second one. Let us consider a two-phase linear programming procedure (see e.g. Cooper, Seiford and Tone, 2000); then for both dual minimization problems the performance measure coincides with the optimal value of the same phase I objective function. Therefore, the problem with the more restricted feasible region will have the higher efficiency score.

\section{Proof of Proposition 2}

Proposition 2 can be proved analogously to Proposition 1 ; when $e_{j_{0}}=0$ and $e_{j} \geq 0$ for all $j$, the constraints of the dual of the input oriented problem equivalent to the DEA problem (3.1)-(3.2) are a subset of the constraints of the dual of the input oriented problem equivalent to the DEA problem (4.2)-(4.3), but the only different constraint (connected to the ethical measure) is redundant, so that the two problems have the same feasible regions.

\section{Proof of Proposition 3}

The proof of Proposition 3 is similar to the proof of Proposition 1 in Banker and Morey (1986a) and rests on the comparison between the feasible region of the dual of the output oriented linear programming problem equivalent to the DEA 
problem (4.2)-(4.3) and the feasible region of the dual of the linear programming problem associated to a convenient transformation of the DEA problem (5.3)-(5.4) (the DEA problem (5.3)-(5.4) can be equivalently written as a problem which minimizes the input to output weighted ratio): the feasible region of the first problem is included in the feasible region of the second one. On the other hand, for both maximization problems the performance measure coincides with the inverse of the optimal value of the dual variable $z_{0}$, so that the problem with the more restricted feasible region will have the higher value of $1 / z_{0}$.

\section{Proof of Proposition 4}

Proposition 4 can be proved analogously to Proposition 3; it is sufficient to note that when $e_{j_{0}}=0$ the constraints which define the feasible regions of the two dual problems that are being compared are identical.

\section{Proof of Proposition 5}

Proposition 5 can be proved by comparing the feasible regions of the dual output oriented linear programming problems (6.6)-(6.7) and (5.1)-(5.2); it suffices to prove the first equality, as the second one follows from Proposition 4 . It can be observed that when $e_{j_{0}}=0$ the constraints which differ between the two problems (connected to the ethical measure) are redundant, so that the feasible regions of the two problems coincide.

\section{Proof of Proposition 6}

To prove the first part of Proposition 6 let us point out that the binary variable $d^{(3)}$ in the multiple categories case takes value 0 for all the ethical funds and value 1 for the non-ethical funds, exactly as the variable $d$ in the binary categorical model, and constraint (6.4) is one of the constraints (6.7) . Hence, the constraints of the binary problem (6.2)-(6.3) are a subset of the constraints of the multiple categories problem (6.6)-(6.7). The inequality (6.9) then follows.

Moreover, for the non-ethical funds all the binary variables have value 1 and the constraints which involve the binary values are redundant. Therefore, when $j_{0}$ is one of the non-ethical funds the two problems have the same feasible regions and the equality (6.10) follows.

\section{References}

Ang, J.S., J.H. Chua. 1979. Composite measures for the evaluation of investment performance. Journal of Financial and Quantitative Analysis 14 361-384.

Banker, R. D., R.C. Morey. 1986a. Efficiency analysis for exogenously fixed inputs and outputs. Operations Research 34 513-521.

Banker, R. D., R.C. Morey. 1986b. The use of categorical variables in Data Envelopment analysis. Management Science 32 1613-1627.

Basso, A., S. Funari. 2001. A data envelopment analysis approach to measure the mutual fund performance. European Journal of Operational Research 135 17-32. 
Boussofiane, A., R.G. Dyson, E. Thanassoulis. 1991. Applied data envelopment analysis, European Journal of Operational Research 52 1-15.

Charnes, A., W.W. Cooper, A.Y. Lewin, L.M. Seiford. 1994. Data envelopment analysis: Theory, methodology, and application, Kluwer Academic Publishers, Boston.

Charnes, A., W.W. Cooper, E. Rhodes. 1978. Measuring the efficiency of decision making units. European Journal of Operational Research 2 429-444.

Charnes, A., W.W. Cooper, E. Rhodes. 1979. Short communication. Measuring the efficiency of decision making units. European Journal of Operational Research 3339.

Cooper, W.W., L.M. Seiford, K. Tone. 2000. Data envelopment analysis: A comprehensive text with models, applications, references and DEA-Solver Software, Kluwer Academic Publishers, Boston.

Jensen, M.C.. 1968. The performance of mutual funds in the period 1945-1964. Journal of Finance 23 389-416.

Levhari, D., H. Levy. 1977. The Capital Asset Pricing Model and the investment horizon. The Review of Economics and Statistics LIX 92-104.

Levy, H.. 1972. Portfolio performance and the investment horizon. Management Science 18 645-653.

Morey, M.R., R.C. Morey. 1999. Mutual fund performance appraisals: a multi-horizon perspective with endogenous benchmarking. Omega 27 241-258.

Murthi, B.P.S., Y.K. Choi, P. Desai. 1997. Efficiency of mutual funds and portfolio performance measurement: A non-parametric approach. European Journal of Operational Research 98 408-418.

Sharpe, W.F.. 1966. Mutual fund performance. Journal of Business 34 119-138.

Treynor, J.L.. 1965. How to rate management of investment funds. Harvard Business Review 43 63-75. 